\title{
Quo vadis BADO? ${ }^{1}$
}

\section{Quo vadis BADO?}

Autor

Institut
Hermann SpießI

Klinik für Psychiatrie, Psychotherapie und Psychosomatik, Bezirkskrankenhaus Landshut

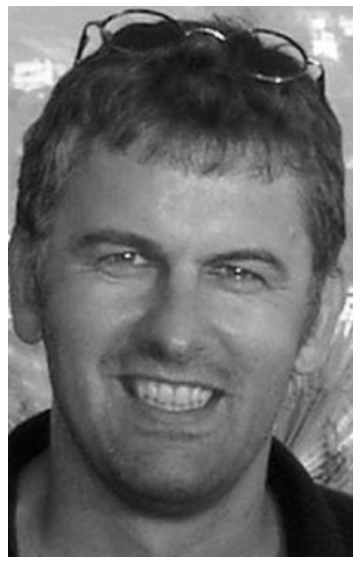

Priv.-Doz. Dr. med. Hermann SpießI
Bibliografie

Dol $10.1055 / \mathrm{s}-0029-1220454$

Psychiat Prax 2009; 36:

305-307

(c) Georg Thieme Verlag KG

Stuttgart . New York.

ISSN 0303-4259

Korrespondenzadresse

Priv.-Doz. Dr. med.

Hermann SpießI

Klinik für Psychiatrie, Psychotherapie und Psychosomatik, Bezirkskrankenhaus Landshut Prof.-Buchner-Straße 22 84034 Landshut

h.spiess|@bkh-landshut.de
Die Basisdokumentation (BADO) für die stationär-psychiatrische Versorgung weist bereits eine über 150-jährige Geschichte auf, beginnend mit dem „Normal-Schema“ von Flemming 1846, die DGPN-BADO von Dilling und Mitarb. 1982 und schließlich die DGPPN-BADO von Cording und Mitarb. 1995. Diese von der DGPPN für die bundesweite Dokumentation in psychiatrisch-psychotherapeutischen Kliniken und Abteilungen empfohlene Form beinhaltet über 70 soziodemografische und erkrankungsbezogene Variablen, die im Routinebetrieb für jeden Patienten erhoben werden [1,2]. Gerade in Zeiten zunehmenden ökonomischen Drucks, geringeren personellen Ressourcen und zunehmenden administrativen Aufgaben [3] ist die DGPPN-BADO unter anhaltender Kritik. In manchen Kliniken für Psychiatrie und Psychotherapie wurde sie auf ein Minimum reduziert, Auswertungen finden oft nicht mehr statt, „Datenfriedhöfe“ entstehen. Auf der anderen Seite werden auch BADO-Daten gerade zur ökonomischen Steuerung von Prozessen verwendet, die methodisch inadäquat ausgewertet und vorschnell interpretiert werden.

Eine Reihe der in der DGPPN-BADO erfassten Variablen sind durchaus als Indikatoren geeignet, die Prozessqualität und die Ergebnisqualität in psychiatrisch-psychotherapeutischen Kliniken und Abteilungen zu beschreiben [1,2]:

- Prozessqualität: diagnostische Maßnahmen, Psychopharmakotherapie, Probleme bei der Psychopharmakotherapie, andere somatische Therapien, Psychotherapie, Probleme bei der Psychotherapie, soziotherapeutische Maßnahmen, Komplikationen: Todesfälle, Suizi-

\footnotetext{
${ }^{1}$ Basierend auf dem Vortrag „Von der BADO zum sektorübergreifenden Datenset“ beim 3. DGPPN-Hauptstadtseminar (Referat Versorgung), Literaturhinweis: Gaebel W, Spießl H, Becker T (Hrsg). Routinedaten in der Psychiatrie. Sektorübergreifende Versorgungsforschung und Qualitätssicherung. Steinkopff Verlag, Darmstadt 2009; $1-7$
}

dalität, Aggression, Fixierung, Entweichung, Entlassung gegen ärztlichen Rat.

- Ergebnisqualität: psychosoziales Funktionsniveau (GAF), Schweregrad der Erkrankung (CGI), soziales Outcome (Arbeit, Wohnung), (kumulierte) Verweildauer, Wiederaufnahmerate.

\section{BADO als Instrument der Versorgungs- forschung und Qualitätssicherung $\nabla$}

Aufgrund fehlender Fallregister in Deutschland ist es ein wesentlicher Vorteil der DGPPN-BADO, dass Auswertungen auf der Basis einer großen Fallzahl (meist mehrere 10000 Patienten), über einen langen Zeitraum (über 10 Jahre) und auch klinikübergreifend erfolgen können. Die in den letzten Jahren durchgeführten Evaluationen erbrachten plausible und klinisch relevante Daten, zeigten aber bei einigen Variablen auch Defizite auf [4]. Die DGPPN-BADO lieferte eine Vielzahl gesundheitspolitisch und -ökonomisch wichtiger Daten zur stationär-psychiatrischen Versorgung in Deutschland, so z. B. zu Verweildauer, Wiederaufnahmerate und Grad der Besserung [5] oder High Utilizer [6], aber auch zu klinisch wichtigen Themen wie z. B. Suizidalität $[7,8]$ oder Aggression [9]. Zahlreiche krankheitsbezogene BADOAuswertungen wie z.B. zu Schizophrenien, Depressionen, bipolaren affektiven Störungen, Intelligenzminderung und deren Behandlung erbrachten plausible Resultate und versorgungsrelevante Erkenntnisse [10-13]. Auch Behandlungswege psychiatrischer Patienten konnten nachgezeichnet und Konsequenzen für die Versorgung abgeleitet werden [14]. Auch umfassende Therapieevaluationen $[15,16]$ und Evaluationen der stationären Versorgung $[17,18]$ fanden statt. 


\section{Limitationen und Revision der DGPPN-BADO $\nabla$}

Seit Einführung der DGPPN-BADO besteht eine Diskussion um die Güte der dort verwendeten Qualitätsindikatoren hinsichtlich ihrer Validität, Reliabilität, Objektivität, Praktikabilität, Transparenz und Patientenorientierung. Daneben und neben den Problemen bei der Implementierung der BADO in ein Klinikinformationssystem (KIS) wird angemerkt, dass keine ereignisbezogene Dokumentation möglich ist [19] und eine personenbezogene, sektorübergreifende Datenerfassung nicht gegeben ist.

Eine Revision der DGPPN-BADO nach nun fast 15 Jahren ihrer Implementierung ist sicher erforderlich. Eine solche Revision sollte auf evidenzbasierten Grundlagen (z.B. Missing-Analysen vorhandener Daten, BADO-Evaluationen, Analysen der Güte der Qualitätsindikatoren, Anwenderbefragungen) und im Vergleich mit anderen „BADOs“ sowie im Hinblick auf Forderungen der Qualitätsberichterstattung und in Bezug zu aktuellen Behandlungsleitlinien erfolgen. Grundzüge einer solchen Revision der DGPPN-BADO könnten sein: deutliche Straffung soziodemografischer und krankheitsbezogener anamnestischer Variablen, genauere Spezifikation des Behandlungsprozesses (insb. Aktualisierung der Items zur Psychopharmakotherapie), Erweiterung der Outcome-Evaluation um eine subjektive Komponente (Lebensqualität, Patientenzufriedenheit) und bessere Operationalisierung der Variablen zum sozialen Outcome (Wohnen, Arbeit, Kontakte).

Ein möglicher Lösungsweg könnte ein modularer Aufbau sein, d.h. neben Basisvariablen („Kernmodul“) werden zusätzliche Module gebildet, z.B.

- abteilungsspezifisch: Sucht, Gerontopsychiatrie,

Psychosomatik, Forensik, ...

- diagnosenspezifisch: Depression, Schizophrenie, Demenz, ...

- therapiebezogen: Psychopharmakotherapie,

Psychotherapie, ...

- ereignisbezogen: Zwangsmaßnahmen, Gewalt,

Suizidalität, ...

Eine Weiterentwicklung des modularen Systems könnte sich durch die Kombination der DGPPN-BADO mit anderen Basisdokumentationen (z. B. AmBADO, BADO-K u. a.) ergeben, wenn sektorübergreifende Kernkriterien definiert werden, die dann modular mit sektorspezifischen Kriterien ergänzt werden. Somit wäre eine personenzentrierte Evaluation sektorübergreifend im Längsschnitt möglich. Diese Weiterentwicklung der DGPPN-BADO würde auch einem neuen Paradigma psychiatrischer Qualitätssicherung [20] besser entsprechen, das neben institutionszentrierten Auswertungen auch personenzentrierte Evaluationen des Langzeit-Outcomes vorsieht und damit den Interessen der Betroffenen wie auch der gesamtgesellschaftlichen Perspektive besser gerecht werden könnte, insbesondere wenn ein flächendeckender Einsatz erfolgt.

\section{Weiterentwicklung zu einem sektorübergreifen- den Datenset \\ $\nabla$}

Dies erfordert aber eine Weiterentwicklung nicht nur der DGPPN-BADO, sondern der gesamten Basisdokumentation im ambulanten, stationären und komplementären Bereich der Psychiatrie und Psychotherapie. Ein guter Ansatz dazu findet sich im sog. Health Care Quality Indicators Project (HCQI) der OECDLänder, in dem in einem strukturierten Review-Prozess nach festgelegten Auswahlkriterien (Bedeutung der Indikatoren für
Versorgung, Wissenschaftlichkeit, Machbarkeit) Indikatoren der Versorgung ausgewählt wurden [21]. Im Ergebnis ergaben sich 12 Indikatoren zur Erfassung der Behandlungskontinuität, der Koordination, der Behandlung und des Behandlungsergebnisses. Diese erfassen z.T. nur sehr grob die Ergebnisqualität (Mortalität bei schwerer seelischer Erkrankung), z.T. sehr speziell die Prozessqualität (Verwendung anticholinerger Antidepressiva bei älteren Patienten). Relevante Indikatoren sind sicherlich z.B. die Zahl der stationären Wiederaufnahmen, die Zahl der ambulanten Kontakte oder die Kontinuität der Behandlung mit Antidepressiva. Diese 12 Indikatoren alleine erscheinen aber nicht ausreichend, die Versorgung psychisch Kranker in den jeweiligen Institutionen, in einer Versorgungsregion oder bundesweit angemessen abzubilden. Selbst ein „Minimum Data Set" wie in England umfassen 60 Variablen und reichen von administrativen Daten bis hin zu Variablen des Behandlungsprozesses im ambulanten und stationären Bereich [22].

Die Entwicklung eines sektorübergreifenden Datensets bedarf sicher einer Herangehensweise wie im HCQI-Projekt und sollte Indikatoren bezüglich sinnvoller Kriterien der Versorgung operationalisieren. Ein Anhalt könnten folgende, schon vor Jahren von der Joint Commission on Accreditation of Healthcare Organization (JCAHO) vorgestellten, qualitätsrelevanten Kriterien der Versorgung sein:

- Zugänglichkeit der Versorgung (accessability)

- Rechtzeitigkeit der Versorgung (timeliness)

- Angemessenheit der Versorgung (appropriateness)

- Kontinuität der Versorgung (continuity)

- Wirksamkeit in der Versorgungspraxis (effectiveness)

- Wirksamkeit unter Idealbedingungen (efficacy)

- Wirtschaftlichkeit der Versorgung (efficiency)

- Patientenorientierung in der Versorgung (patient perspective)

- Sicherheit in der Versorgungsumgebung (safety)

\section{Resümee und Ausblick}

$\nabla$

Als Resümee lässt sich ziehen, dass die DGPPN-BADO in den letzten Jahren eine unverzichtbare Datengrundlage für Qualitätssicherung, Versorgungsforschung und Klinikmanagement darstellte. Eine Weiterentwicklung erscheint notwendig und sollte den Erfordernissen einer sektorübergreifenden Dokumentation gerecht werden.

Vor diesem Hintergrund fand am 17. Juni 2008 in Berlin ein initiales DGPPN-Hauptstadtseminar zum Thema „Routine-Daten in der Psychiatrie“ statt, deren Beiträge in Buchform erschienen sind [23]. Die Beiträge verdeutlichen, dass viel Arbeit zu Routinedaten in Psychiatrie und Psychotherapie geleistet worden ist. Verschiedene Instrumente kommen in unterschiedlichen Segmenten des Versorgungssystems zum Einsatz, eine Reihe wissenschaftlicher Arbeiten sind auf der Grundlage von BADO-Daten entstanden, Routinedaten werden in der klinischen Berichterstattung und in Qualitätsberichten verwendet. Verschiedene konzeptuelle Ansätze wie die sektorübergreifende Dokumentation, die Leitlinienkonformität der Versorgung, die Entwicklung von Qualitätsindikatoren und der Begriff der Personenzentrierung wurden entwickelt. Noch fehlt jedoch ein Gesamtkonzept zur Verwendung von Routinedaten, welches die Anforderungen der Machbarkeit, hinreichender Differenziertheit und einer klaren Ziel- oder Leitlinienorientierung erfüllt. Neue Aspekte ergeben sich zudem durch die Einführung eines pauschalierenden 
Entgeltsystems, das - obwohl auf der Grundlage der PsychPVKlassifikation entwickelt - weitere Ansprüche an die Güte der Dokumentation im Fachgebiet Psychiatrie und Psychotherapie stellen wird.

Wie kann es weitergehen? In einem strukturierten Review-Prozess sollten unter Berücksichtigung der o.g. internationalen Entwicklungen und Erfahrungen (z. B. auch SIEP-DIRECT Programm, [24]) die für unser Versorgungssystem relevanten Indikatoren nach festgelegten Auswahlkriterien wie Bedeutung für Versorgung, Leitlinienorientierung, Wissenschaftlichkeit und Machbarkeit ausgewählt werden. Ein Konsens der beteiligten Institutionen, Kostenträger und Fachgesellschaften erscheint dabei ebenso wichtig wie die Berücksichtigung der Anliegen von Patienten und Angehörigen [25,26]. Die Entwicklung eines modularen, personenbezogenen und sektorübergreifenden Datensets, das geeignet ist, die Versorgung psychisch Kranker in Deutschland abzubilden, relevante Veränderungen sensitiv erfasst und eine valide Datengrundlage in der gesundheitspolitischen Diskussion liefert, ist das Ziel der Zukunft. Von der Versorgungsforschung muss dieser Weg begleitet werden [27-29].

\section{Literatur}

1 Cording C, Gaebel W, Spengler A et al. unter Mitarbeit von Spindler P und Krischker S. Die neue psychiatrische Basisdokumentation. Eine Empfehlung der DGPPN zur Qualitätssicherung im (teil-)stationären Bereich. Spektrum der Psychiatrie und Nervenheilkunde 1995; 24: 3-41

2 Hübner-Liebermann B, Spießl H, Spindler P et al. Verbesserte Erfassung des Behandlungsprozesses mit einer modifizierten BADO. Krankenhauspsychiatrie 2000; 11: 102-104

3 Putzhammer A, Senft I, Fleischmann $H$ et al. Eine Tätigkeitsanalyse in psychiatrischen Versorgungskliniken. Nervenarzt 2006; 77: 372-384

4 Spießl H, Binder H, Hübner-Liebermann B et al. Wie gut ist die psychiatrische Basisdokumentation, was kann sie leisten und brauchen wir sie überhaupt? Nervenarzt 2005; 76: S357-358

5 Spießl H, Binder H, Cording C et al. Klinikpsychiatrie unter ökonomischem Druck. Dt Ärztebl 2006; 103: 2549-2552

6 Spießl H, Hübner-Liebermann B, Binder $H$ et al. „Heavy Users“ in einer psychiatrischen Klinik - Eine Kohortenstudie mit 1811 Patienten über fünf Jahre. Psychiat Prax 2002; 29: 350-354

7 Neuner T, Schmid $R$, Wolfersdorf $M$ et al. Predicting inpatient suicides and suicide attempts by using clinical routine data? Gen Hosp Psychiatry $2008 ; 30$ : 324-330

8 Spießl H, Hübner-Liebermann B, Cording C. Suicidal behaviour of psychiatric in-patients. Acta Psychiatr Scand 2002; 106: 134-138

9 Spießl H, Krischker S, Cording C. Aggressive Handlungen im psychiatrischen Krankenhaus. Psychiat Prax 1998; 25: 227-230
10 Hübner-Liebermann B, Spießl H, Cording C et al. Psychopharmakotherapie depressiver Patienten im Vorfeld der stationären Aufnahme. Psychopharmakotherapie 2006; 13: 147-153

11 Hübner-Liebermann B, Spießl H, Cording C et al. Psychopharmakotherapie schizophrener Patienten im Vorfeld der stationären Aufnahme. Psychopharmakotherapie 2007; 14: 116-121

12 Spießl A, Binder $H$, Cording $C$ et al. Patienten mit Intelligenzminderung in der psychiatrischen Klinik. Psychiat Prax 2008; 35: 67-72

13 Spießl H, Hübner-Liebermann B, Cording $C$. Unterschiede zwischen unipolaren und bipolaren affektiven Störungen. Fortschr Neurol Psychiat 2002; 70: 403-409

14 Hübner-Liebermann B, Spießl H. Wer kommt woher, wer geht wohin? Behandlungspfade psychiatrischer Patienten. Nervenarzt 2003; 74: S66

15 Spießl H, Hübner-Liebermann B, Schmid $R$ et al. Psychotherapie in der stationär-psychiatrischen Versorgung. Psychotherapie 2008; 13: 50-55

16 Wetterling T, Junghanns K, Müßigbrodt $H$ et al. Erfassung der Therapieergebnisse im Rahmen der Qualitätssicherung in einer psychiatrischen Klinik. Nervenarzt 1997; 68: 742-751

17 Kipp J, Kristen R, Kunze $H$ et al. Basisdokumentation: Die stationäre Versorgung einer Region durch ein Psychiatrisches Krankenhaus und eine psychiatrische Abteilung. Nervenarzt 1998; 69: 782-790

18 Hübner-Liebermann B, Hajak G, Spießl H. Versorgungsepidemiologie: Entwicklung in der stationär-psychiatrischen Versorgung 1996-2006. Psychiat Prax 2008; 35: 387-391

19 Fähndrich E. Erfahrungen mit der Basisdokumentation im klinischen Bereich. Psycho 2000; 26: 381-385

20 Cording C. Plädoyer für ein neues Paradigma psychiatrischer Qualitätssicherung. Psychiat Prax 2003; 30: 225-229

21 www.oecd.org/dataoecd/28/32/33865630.pdf

22 Glover GR. A comprehensive clinical dataset for mental health care in England. Soc Psychiatry Psychiatr Epidemiol 2000; 35: 523-529

23 Gaebel W, Spießl H, Becker T, Hrsg. Routinedaten in der Psychiatrie. Sektorübergreifende Versorgungsforschung und Qualitätssicherung. Darmstadt: Steinkopff Verlag, 2009

24 Ruggeri M. Guidelines for treating mental illness: love them, hate them. Can the SIEP-DIRECT'S Project serve in the search for a happy medium? Epidemiologia e Psichiatria Sociale 2008; 17: 270-277

25 Kühn-Mengel $H$. Ausbau der Patientenberatung: bei psychischen Erkrankungen von besonderer Bedeutung. Psychiat Prax 2008; 35 (3): 107

26 Reichert T, Kissling W, Scheuring $E$ et al. Patientenbeteiligung in der Psychiatrie - eine kritische Bestandsaufnahme. Psychiat Prax 2008; 35 (3): 111-121

27 Riedel-Heller SG, Bramesfeld A, Roick C et al. Der Ruf nach mehr Versorgungsforschung. Psychiat Prax 2008; 35 (4): 157-159

28 Bramesfeld A, Riedel-Heller SG. Prioritäre Themen der Versorgungsforschung zur psychischen Gesundheit. Psychiat Prax 2008; 35 (7): 315-317

29 Hausner H, Hajak G, Spießl H. Verliert die Soziotherapie im wissenschaftlichen Diskurs an Bedeutung? Psychiat Prax 2007; 35(8): 395-399 